\title{
Systemic taurine treatment provides neuroprotection against retinal photoreceptor degeneration and visual function impairments
}

This article was published in the following Dove Press journal:

Drug Design, Development and Therapy

\author{
Ye Tao ${ }^{1,2, *}$ \\ Miao $\mathrm{He}^{3, *}$ \\ Qinghua Yang' \\ Zhao $\mathrm{Ma}^{3}$ \\ Yingxin Qu' \\ Wen Chen $^{3}$ \\ Guanghua Peng ' \\ Dengke Teng ${ }^{4}$ \\ 'Department of Physiology, Basic Medical \\ College, Zhengzhou University, \\ Zhengzhou 45000I, People's Republic of \\ China; ${ }^{2}$ Lab of Visual Cell Differentiation, \\ Basic Medical College, Zhengzhou \\ University, Zhengzhou 45000I, People's \\ Republic of China; ${ }^{3}$ Department of \\ Neurosurgery, Central Hospital of \\ Wuhan, Tongji Medical College, \\ Huazhong University of Science and \\ Technology, Wuhan 4300I4, People's \\ Republic of China; ${ }^{4}$ Department of \\ Ultrasound, China-Japan Union Hospital \\ of Jilin University, Changchun, People's \\ Republic of China
}

*These authors contributed equally to this work

Correspondence: Dengke Teng Department of Ultrasound, China-Japan Union Hospital of Jilin University, Changchun, People's Republic of China Email drdengketeng@।63.com

Wen Chen

Department of Neurosurgery, Central Hospital of Wuhan, Tongji Medical College, Huazhong University of Science and Technology, Wu Hang 4300I4,

People's Republic of China

Email drwenchen@163.com
Objective: Retinitis pigmentosa causes progressive photoreceptor degeneration in the subjects while no clinical therapy exists. The present study sought to evaluate the potential protective effects of taurine on a pharmacologically induced RP animal model.

Methods: Photoreceptor degeneration in mice was induced by an intraperitoneal injection of $\mathrm{N}$-methyl-N-nitrosourea (MNU). The MNU-administrated mouse received taurine treatment and then they were examined by electroretinography, spectral-domain optical coherence tomography, optokinetic test, and histological and immunohistochemistry assay.

Results: Prominent taurine deficiency was found in the retinas of MNU-administered mice. Intravenous taurine treatment increased significantly the retinal taurine level. Morphological studies showed that taurine could alleviate the retinal disorganizations in the MNU-induced mice. Taurine also ameliorated the visual impairments in the MNU-induced mice as evidenced by functional examinations. Immunostaining experiments demonstrated that both the $\mathrm{M}$-cone and S-cone populations in the degenerative retinas are rescued by taurine. In particular, the M-cone photoreceptors in superior-temporal quadrant and the S-cone photoreceptors in inferior-nasal quadrant were preferentially rescued. Mechanism study showed that the photoreceptor apoptosis and oxidative stress in the degenerative retina were effectively alleviated by taurine treatment.

Conclusion: Taurine is protective against the MNU-induced photoreceptor degeneration. Systemic taurine administration may act as a promising therapeutic potion for retinopathies with chronic cycle.

Keywords: neural degeneration, retina, therapeutics

\section{Introduction}

Retinitis pigmentosa (RP) is a family of hereditary retinal dystrophies that is characterized by the progressive night blindness and visual field loss. ${ }^{1,2}$ Photoreceptor degeneration is considered as the key pathogenesis for the irreversible visual impairments in RP patients. Thus far, the specific molecular basis underlying photoreceptor degeneration remains enigmatic. A broad spectrum of genetic defects affecting the retinal structure, metabolism, and phototransduction cycle could eventually lead to photoreceptor degeneration. ${ }^{3}$ The enormous heterogeneity imposes overwhelming challenges for emerging therapeutic strategies. In the past decades, remarkable progress has been made in developing therapeutic strategies such as the gene rectification, stem cell transplantation, and visual prostheses. However, the effects of these treatments are far from satisfactory. RP is still considered as an "incurable" 
retinopathy. ${ }^{4}$ In laboratory work, animal models afford valuable tools for pathologic investigations and therapeutic trials. The N-methyl-N-nitrosourea (MNU)-administered animal is a pharmacologically induced RP model with rapid progressive dynamics. ${ }^{5}$ After a single systemic administration, MNU toxicity induces massive photoreceptor apoptosis in subjects. ${ }^{6}$ The hallmarks of MNU-administered animals, such as the extinguished electroretinogram (ERG) response, disorganized retinal architecture, activated retinal gliosis, and neuronal plasticity, are compared well with these occur in RP patients. ${ }^{7,8}$ Hitherto, the MNU-administered animals have been extensively used to study RP. It is widely accepted that photoreceptor apoptosis acts as the primary pathological events of in RP pathogenesis. ${ }^{9}$ The apoptotic activation is closely associated with oxidative stress during retina degenration. ${ }^{10}$ Photoreceptors are extremely sensitive to oxidative insults owing to their vigorous metabolism and abundant polyunsaturated fatty acids in cellular membranes. Excessive reactive oxygen species (ROS) can perturb redox metabolism, alter mitochondrial membrane permeability, and induce cytochrome c leakage. In particular, the cytochrome c leakage from the mitochondrial is defined as a pivotal initiator for the caspase-mediated apoptotic cascades. ${ }^{11}$ Therefore, targeting the oxidative stress may afford a practical therapeutic strategy against photoreceptor apoptosis.

As a naturally occurring free amino acid, Taurine is enriched in various excitable tissues, such as retina, brain, skeletal, and cardiac muscles. ${ }^{12}$ Taurine carries out a wide spectrum of physiological functions including modulating development, maintaining homeostasis, and stabilizing membrane permeability. Moreover, taurine is recognized as a potent cytoprotective agent because it rescues neurons and cardiomyocytes from hypoxia and ischemic insults. ${ }^{13}$ Several pioneering studies also show that taurine exerts neurotrophic effects on the retinal ganglion cells (RGC). Taurine supplements could prevent RGC degeneration in glaucoma animal model and purified cell cultures. ${ }^{14}$ Enriched taurine nutrition promotes RGC survival in the $\mathrm{P} 23 \mathrm{H}$ rats. ${ }^{15}$ These findings suggest that taurine can positively interfere with RGC death under different pathological conditions. Quantitative analysis of the ocular tissue extracts showed that the taurine concentration forms a gradient across different retinal layers. Taurine was most abundant in the distal layers of retina, including the outer nuclear layer (ONL) and retinal pigment epithelium (RPE) layer. ${ }^{14,16}$ Photoreceptors rank among the cells with exceptionally high level of taurine. Generally, photoreceptors require an adequate supply of extracellular taurine. RPE and Müller cells take up taurine from systemic circulation and pass it on to photoreceptors. Animals would experience severe retinopathy and visual impairments when they are deprived of dietary taurine. ${ }^{14,17,18}$ Hitherto, previous studies have not unequivocally confirmed the taurine-induced effects against photoreceptor degeneration.

In this study, we demonstrated that intravenous delivery increased significantly the plasmatic and retinal taurine level in the MNU-administered mice. As evidenced by the functional and morphological examinations, taurine treatment could rescue the photoreceptors and ameliorate the visual impairments in this RP animal model with rapid progressive dynamics. Mechanism study showed that the photoreceptor apoptosis and oxidative stress were effectively alleviated by taurine treatment. Further clinical studies are necessary to verify these protective effects in RP patients.

\section{Materials and methods}

\section{Animals and study design}

C57BL/6 mice (8-week-old with both sexes) were used and handled according to the statements of the Association for Research in Vision and Ophthalmology (ARVO) for the use of animals. Study protocol was reviewed and approved by the institutional animal care and use committee of the PLA General Hospital. All animals were housed in the airconditioned facility (room temperature: $18-23^{\circ} \mathrm{C}$, humidity: 40-60\%, under 12/12-h light/dark cycle; standard diet and water ad libitum). Experimental animals were divided randomly into four groups: 1) Normal control group: mice were left without any pharmacological administration; 2) MNU group: mice received an intraperitoneal injection of MNU (60 mg/kg; Sigma-Aldrich, MO, USA; Figure 1A); 3) MNU+taurine group: mice received taurine treatment (200 mg/kg; Sigma-Aldrich, MO, USA) via caudal vein injection, once daily for consecutive 7 days before and after MNU administration; 4) MNU+vehicle group: mice received intravenous injection of vehicle (phosphate buffer saline, PBS) once daily for consecutive 7 days before and after MNU administration. 4) Normal+taurine group: mice were administrated with taurine via tail intravenous injection, once daily for consecutive 7 days. Figure $1 \mathrm{~B}$ is a schematic illustration of the experimental protocols.

\section{Optokinetic test}

One week after MNU administration, mice were subjected to optokinetic test. Mice were placed on a platform of the 
A

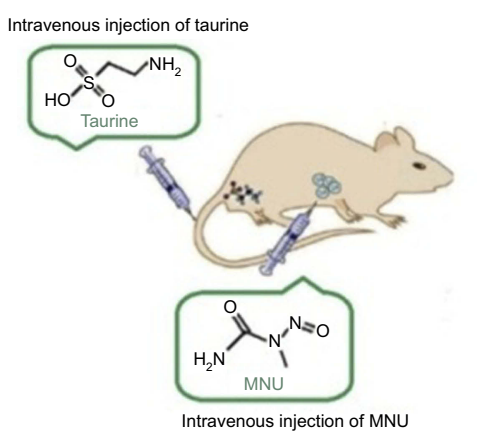

C

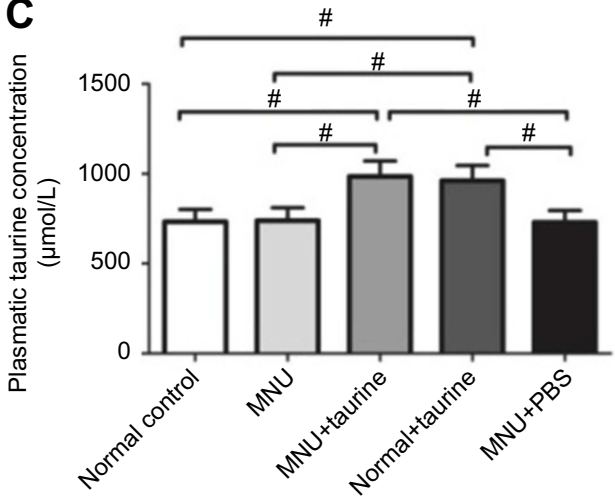

B

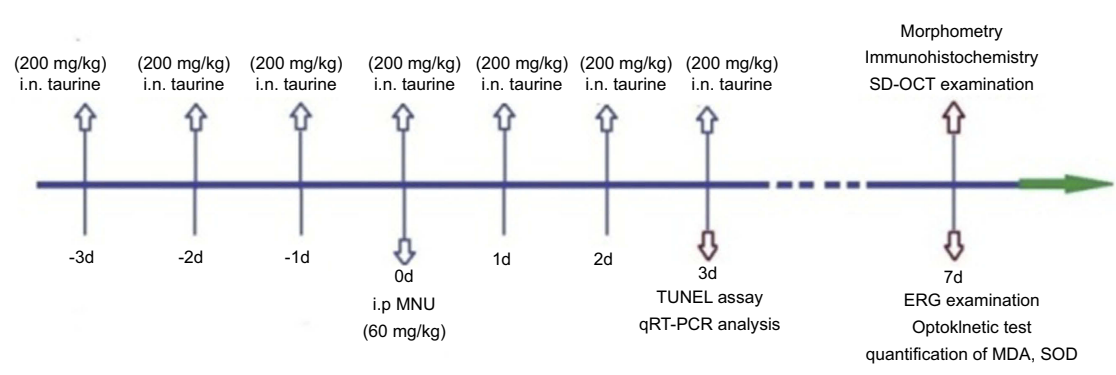

Figure I (A) Photoreceptor degeneration was induced by an intraperitoneal administration of N-methyl-N-nitrosourea (MNU). The MNU-administered mice received taurine treatment via tail intravenous injection, once daily for consecutive 7 days. (B) The schematic illustration of the experimental protocols. (C) The plasmatic taurine concentrations in the Control+taurine and MNU+taurine group were both significantly higher than that in the normal controls. (D) The retinal taurine concentration in the MNU group was significantly lower than that in the normal controls. The retinal taurine concentrations in the MNU+taurine group and Control+taurine group were both significantly higher compared with the normal control group, suggesting that the intravenous injection enhances the taurine level in retina (ANOVA analysis followed by Bonferroni's post-hoc analysis was performed, $* P<0.05,{ }^{\#} P<0.01$, for differences between groups; $n=10$ ).

test box. ${ }^{19}$ Virtual cylinders were projected on the wall of the box, and they turned either in clockwise or counterclockwise direction for the mouse to track. The stepwise functions for correct track responses were used to determine the response thresholds. The initial stimulus in visual acuity measurements was set as $0.200 \mathrm{cyc} / \mathrm{deg}$ sinusoidal pattern with a fixed $100 \%$ contrast. The initial pattern in contrast sensitivity measurements was set as $100 \%$ contrast, with a fixed spatial frequency of $0.128 \mathrm{cyc} / \mathrm{deg}$. All patterns were presented at a speed of 12 degrees/s with the mean luminance of $70 \mathrm{~cd} / \mathrm{m}^{2}$.

\section{Electroretinogram (ERG) examination}

One week after MNU administration, the mice were anesthetized with an intraperitoneal injection of ketamine $(80 \mathrm{mg} / \mathrm{kg})$ and chlorpromazine $(15 \mathrm{mg} / \mathrm{kg}$, Shengda Animal Pharmaceutical, Jilin, China). Their pupils were dilated with $1 \%$ atropine and $2.5 \%$ phenylephrine hydrochloride eye drops (Xing Qi, Shenyang, China), and their eye maintained open during the whole recording process. The RETIport system (Roland Consult, Germany) with custom-made chloride silver electrodes was used for recording. Light stimulations $\left(3.0 \mathrm{~cd} \cdot \mathrm{s} / \mathrm{m}^{2}\right)$ were delivered from a Ganzfeld integrating sphere to stimulate the response. Flash responses were recorded through corneal electrodes, with the reference electrode placed in the subcutaneous space of the cheek, and the neutral electrode clipped to the tail. Recorded signals were filtered with high- and low-pass filters at 100 and $300 \mathrm{~Hz}$, respectively. Totally 60 photopic responses and 10 scotopic responses were collected and averaged for b-waves analysis. Amplitude of a-wave was defined as the distance from baseline to a-wave trough, while the amplitude of b-wave was defined as the distance between trough and peak of each waveform.

\section{Spectral-domain optical coherence tomography (SD-OCT)}

Immediately after ERG examination, mice were transferred to the recording plane of an ultrahigh-resolution instrument (Bioptigen, Durham, NC, USA) when they were still anesthetized. A corresponding box was centered 
on the optic nerve head and eight measurements at the same distance $(3 \mathrm{~mm})$ from each other were executed. Three hundred linear B-scans were obtained, and 30 averaged images were captured to achieve a better resolution. The SD-OCT cross-sectional images were analyzed subsequently with the In-VivoVueTM DIVER 2.4 software (Bioptigen, Durham, NC, USA).

\section{Morphometry and immunohistochemistry}

One week after MNU administration, mice were sacrificed and their eyes were enucleated. The eyecups were immersed in a fixative solution overnight at $4^{\circ} \mathrm{C}$. The anterior segments of eyeballs were cut off without disturbing the retinal tissues. Then, the retinas were embedded in paraffin wax and sectioned in the sagittal plane. Retinal sections (thickness of $5 \mu \mathrm{m}$ ) were stained with hematoxylin and eosin (HE) and examined under light microscopy. For the preparation of retinal flat mount, we first removed the optic nerve head and then separated the sensory retina from the eyecup. Subsequently, retinal specimens were rinsed in $0.01 \mathrm{M} \mathrm{PBS}$, permeabilized in $0.3 \%$ Triton $\mathrm{X}-100$, and blocked in 3\% bovine serum albumin (BSA) for $1 \mathrm{hr}$ at room temperature. Primary antibodies were directed against S-cone opsin or M-cone opsin (1:400, Millipore, MA, USA) or anti-caspase-3 (1:200, Santa Cruz, CA, USA) and were diluted in the incubated solution. Retinal specimens were rinsed with PBS and then incubated with the MTP2 or Cy3-conjugated anti-rabbit IgG (1: 400, Jackson ImmunoResearch Laboratories, USA) for $1 \mathrm{hr}$. Outer segments of cone photoreceptors were stained with a peanut agglutinin conjugated to Alexa Fluor 488 (PNA, 1: 200, L21409, Invitrogen, USA) overnight at $4^{\circ} \mathrm{C}$. Cell nuclei were labeled by incubating the retinal specimen with $10 \mu \mathrm{g} / \mathrm{mL} 4^{\prime}, 6$-diamidino -2-phenylindole (DAPI, Sigma, MA, USA) for $1 \mathrm{hr}$ at room temperature. Retinal specimens were rinsed and mounted with anti-fade vectashield mounting medium (Vector Labs, Burlingame, CA, USA). Fluorescence was analyzed with the Zeiss LSM 510 META microscope (Zeiss, Thornwood, NY, USA) fitted with Axiovision Rel. version 4.6 software (Carl Zeiss AG Manufacturing company, Oberkochen, Germany). All fluorescent images were captured using identical exposure settings. The cone number of four $420 \times 420 \mu \mathrm{m}$ squares which located $1 \mathrm{~mm}$ dorsal, temporal, ventral, and nasal to the center of the optic nerve were determined.

\section{TUNEL assay}

Three days after MNU administration, Terminal deoxyuridine triphosphate nick-end labeling (TUNEL) assay was performed on retinal sections. An in situ cell death detection POD Kit (Roche, Mannheim Germany) was used according to manufacturer's protocol. TUNEL sections were counterstained with DAPI, mounted on slides, and then visualized with confocal microscopy (LSM510, Zeiss, Oberkochen, Germany). Apoptotic index (AI) of the ONL was calculated on the basis of cell numbers (number of TUNEL-positive nuclei/total number of photoreceptor cell nucleix100).

\section{Quantitative reverse}

\section{transcription-polymerase chain reaction (qRT-PCR)}

The mRNA transcripts levels of Bax, Bcl-2, Caspase-3, and Calpain-2 in the retinas were measured by real-time RT-PCR three days after MNU administration. Total RNA was isolated from mouse retinal tissues, and cDNA was synthesized using $\mu \mathrm{MACS}^{\mathrm{TM}}$ DNA Synthesis kit (Miltenyi Biotech $\mathrm{GmbH}$, Bergisch-Gladbach, Germany). All quantitative PCR reactions were performed via a real-time CFX96 Touch PCR detection system (Bio-RadLaboratories, Reinach, Switzerland). The primers used in qRT-PCR were: Bax: 5'-AGCTCTGAACAGATCATGAAGACA-3' (forward) and 5'-CTCCATGTTGTTGTCCAGTTCATC-3' (reverse); Bcl-2: 5'-GGACAACATCGCTCTGTGGATG A-3' (forward) and 5'-CAGAGACAGCCAGGAGAAATCA A-3' (reverse); Caspase-3: 5'-TGTCGATGCAGCTAACC-3' (forward) and 5'-GGCCTCCACTGGTATCTTCTG-3' (reverse); Calpain-2: 5'-CCCCAGTTCATTATTGGAGG-3' (forward) and 5'-GCCAGGATTTCCTCATTCAA-3' (reverse). The relative expression levels were normalized and quantified to obtain the $\Delta \Delta \mathrm{CT}$ values (DATA assist Software v2.2, Applied Biosystems, Foster City, CA, USA).

\section{Measurements of taurine level}

Blood samples were collected from caudal vein and stored in hemolysis tubes containing heparin (14 IU/mL). Subsequently, the blood samples were centrifuged at 3,000 $\mathrm{g}$ for 15 mins. Ion exchange chromatography was performed to evaluate the plasmatic amino-acid analysis using Jeol AminoTac analyzer (JLC-500, Jeol Ltd., Tokyo, Japan). For retinal taurine concentration assessment, mice were sacrificed and their eyes were enucleated. Retina was gently removed from the sclera shell and was homogenized in 300 
$\mu \mathrm{L} 0.4 \mathrm{M}$-potassium borate buffer and $50 \mu \mathrm{L} 20 \%$ sulfosalicylic acid. Bruker Avance III spectrometer (Bucker BioSpin, Billerica, MA, USA) was used to record the Highresolution magic angle spinning (HRMAS) spectra following a previously described method. Taurine concentration was quantified by the method of the external standard and was expressed as $\mathrm{nmol} / \mathrm{mg}$ protein.

\section{Determination of malondialdehyde (MDA) and superoxide dismutase (SOD) levels}

SOD activity was examined with the SOD Assay Kit-WST (Jiancheng Biotech Ltd., Nanjing, China). One unit (U) of SOD activity was defined as the amount of enzyme causing half inhibition in the nitroblue tetrazolium reduction rate. SOD activity was expressed as units/mg protein. The concentration of malondialdehyde (MDA) was assessed using total bile acids colorimetric assay under the guidance of the manufacturer's protocol (Jiancheng Biotech Ltd., Nanjing, China). The concentration of MDA was expressed as $\mathrm{nmol} / \mathrm{g}$ retinal protein.

\section{Statistical analysis}

Statistical difference between the animal groups was processed using the ANOVA analysis followed by Bonferroni's post-hoc analysis. $P$-value less than 0.05 was considered significant. All the values are presented as mean \pm standard deviation (SD).

\section{Results}

\section{Plasmatic and retinal taurine level in mice}

No clinical sign or system symptom was evident in any of the experimental animals during the experimental process. Plasma specimens were collected from animals and were measured for taurine concentration. The plasmatic taurine concentration in the MNU group was not significantly different from that in the normal control group $(P>0.05, \mathrm{n}=10)$, suggesting that MNU administration would not affect the plasmatic taurine level in normal mice. Of note, the plasmatic taurine concentration in the Control+taurine group was significantly higher than that in the normal control group $(P<0.01, \mathrm{n}=10$; Figure 1C). The plasmatic taurine concentration in the $\mathrm{MNU}+$ taurine group was significantly higher than that in the MNU group $(P<0.01, \mathrm{n}=10)$. As the plasmatic taurine concentration can be affected by endogenous amino acid release from the other tissue (eg, liver, brain, and muscle), the retinal taurine level might act as a more reliable indicator to reveal the efficiency of taurine administration. The retinal taurine concentration in the MNU group was significantly lower than that in the normal control group $(P<0.01, \mathrm{n}=10$; Figure 1D), suggesting that the MNU toxicity induced taurine deficiency in mice retinas. However, a prominent increase in retinal taurine level was found in the taurine-administrated mice. The retinal taurine concentrations in the MNU+taurine group and Control+taurine group were both significantly higher compared with the normal control group $(P<0.01, \mathrm{n}=10)$, suggesting that the intravenous injection enhances efficiently the taurine level in retina.

\section{Taurine mediated protective effects on retinal morphology}

OCT examination was performed to analyze the retinal structure in vivo. Differences in retinal thickness are clearly visible in the representative imagines from each animal group (Figure 2A). The ONL in the retinas of the MNU group was terribly disrupted. On the other hand, the mice in the MNU+taurine-administered group had relatively intact retinal architectures. The retinal thickness in the MNU +taurine group was significantly larger than that in the MNU group $(P<0.01 ; \mathrm{n}=10)$, suggesting the taurine treatment could preserve the retinal structure effectively. The retinal thickness in the MNU+PBS group was not different from that in MNU group $(P>0.05, \mathrm{n}=10)$. On closer inspection, the retinal sections were visualized microscopically. The retinas in the normal control group were highly organized, with intact layers. The overall retinal morphology was also intact in the retinas of Normal+taurine group. By contrast, the ONL was not discernible in the retinas of MNU group (Figure 2B). Comparison analysis showed that the mean ONL thickness of the MNU+taurine group was significantly larger than that in the MNU group $(P<0.01$; $\mathrm{n}=10)$. The mice in the MNU+taurine group maintained a substantial proportion $(\sim 62 \%)$ of ONL. The mean ONL thickness in the MNU+PBS group was not different from that in MNU group $(P>0.05, \mathrm{n}=10)$. Additionally, the mean ONL thickness of the Control+taurine group was not significantly different from that in the normal control group $(P>0.05 ; \mathrm{n}=10)$, suggesting the taurine treatment would not affect the photoreceptor vitality in normal mice.

Moreover, TUNEL assay was performed to examine the apoptotic status of retina. Nuclei labeled by the TUNEL staining were rarely detected in the retinas of normal control group (Figure 2C). Conversely, numerous 
A

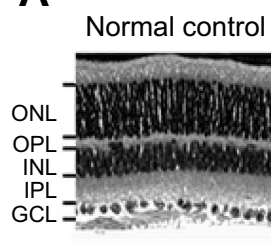

B
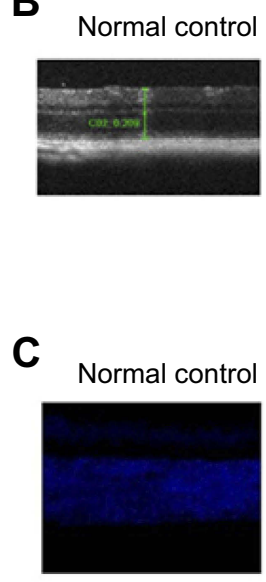
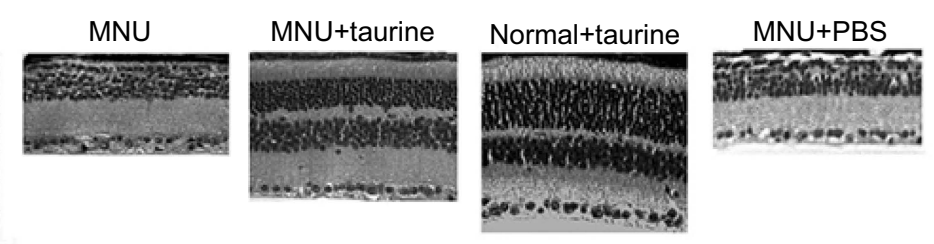

MNU
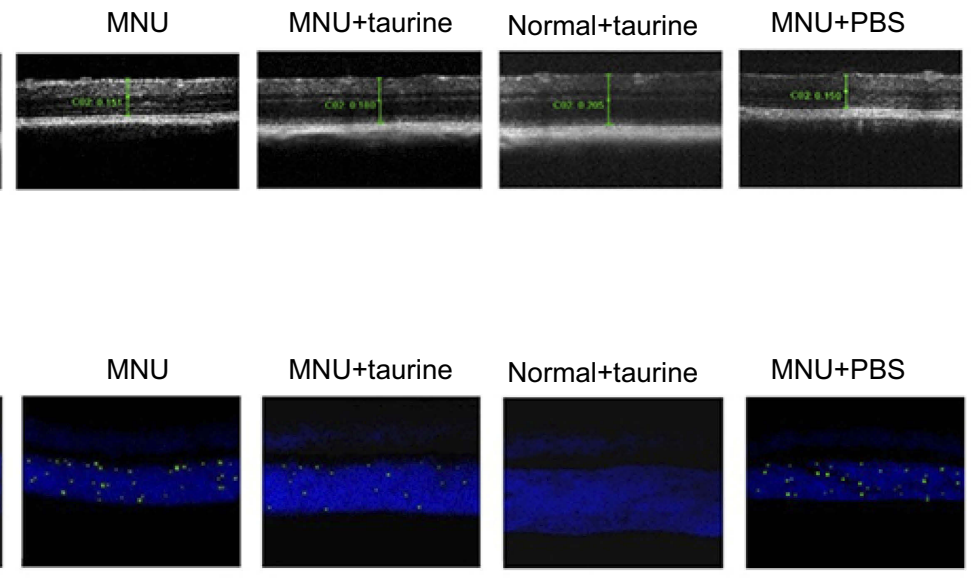
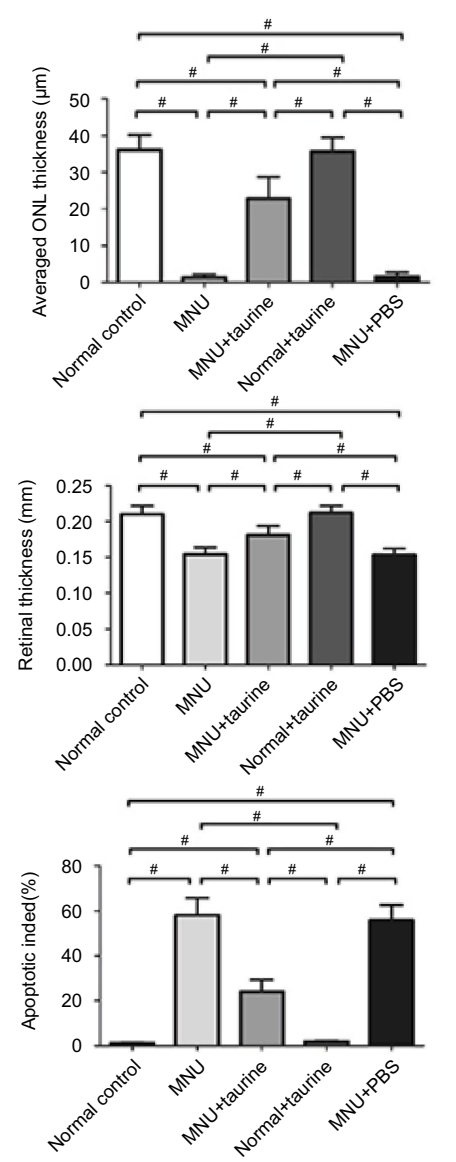

Figure 2 (A) As shown in the OCT images, the ONL in the retinas of the N-methyl-N-nitrosourea (MNU) group was terribly disrupted. The mice of the MNU+taurineadministered group had relatively intact retinal architectures. The retinal thickness in the MNU+taurine group was significantly larger than that in the MNU group, suggesting the taurine treatment could preserve the retinal structure effectively. (B) The ONL was not discernible in the retina sections of MNU group. The mean ONL thickness of the MNU+taurine group was significantly larger than that in the MNU group. (C) Numerous TUNEL-labeled cells were found in the retinas of the MNU group. TUNELlabeled cells in the MNU+taurine group were remarkably less compared with the MNU group. The Al of the MNU+taurine group was significantly smaller compared with the MNU group (ANOVA analysis followed by Bonferroni's post-hoc analysis was performed, ${ }^{\#} P<0.01$, for differences between groups; $n=10$ ).

Abbreviations: GCL, Ganglion cell layer; IPL, inner plexiform layer; OPL, outer plexiform layer; ONL, outer nuclear layer; INL, inner nuclear layer.

TUNEL-labeled cells were found in the retinas of the MNU group, suggesting that MNU toxicity induced massive photoreceptor apoptosis. The TUNEL-labeled cells in the MNU+taurine group were remarkably less compared with the MNU group. Quantification analysis suggested that the AI of the MNU group was significantly larger compared with the normal control group $(P<0.01 ; \mathrm{n}=10)$. On the other hand, the AI of the MNU+taurine group was significantly smaller compared with the MNU group $(P<0.01 ; \mathrm{n}=10)$. These data suggested that the taurine could suppress the photoreceptor apoptosis in MNUadministered mice.

\section{Taurine mediated protective effects on visual function}

Representative ERG waveforms of different animal groups are shown in Figure 3A. Typical ERG responses were evident in the normal control group. The stimulus also induced regular ERG responses in the normal+taurine group. The scotopic and photopic a-wave amplitudes in the normal+taurine group were not significantly different from those in the normal control group $(P>0.05 ; \mathrm{n}=10$; Figure $3 \mathrm{~B}$ and $\mathrm{C})$. The scotopic and photopic a-wave amplitudes in the MNU+taurine group were significantly larger than those in the MNU group $(P<0.01 ; \mathrm{n}=10)$. Moreover, the scotopic and photopic b-wave amplitudes in the normal+taurine group were not significantly different from those in the normal control group $(P>0.05 ; \mathrm{n}=10$; Figure $3 \mathrm{D}$ and $\mathrm{E})$. The ERG responses in the MNU group were terribly abolished. Both the scotopic and scotopic b-wave amplitudes in the MNU group were significantly smaller compared with the normal control group $(P<0.01 ; \mathrm{n}=10)$. The amplitudes of ERG b-waves in the MNU+PBS group were not significantly different from those in the MNU group $(P>0.05$, 
A

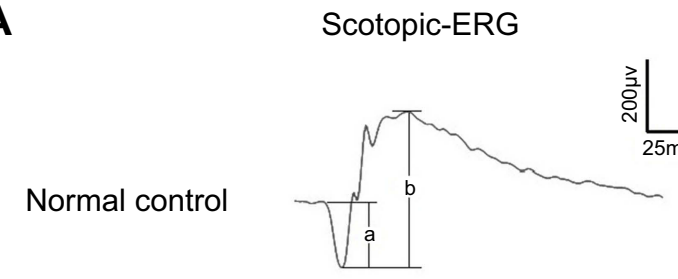

MNU

MNU+taurine

Normal+taurine
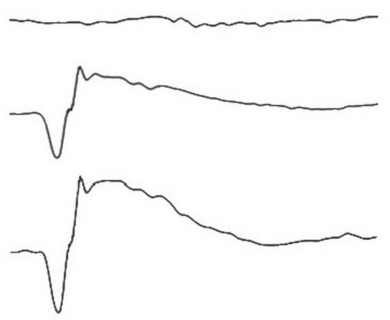

Photopic-ERG
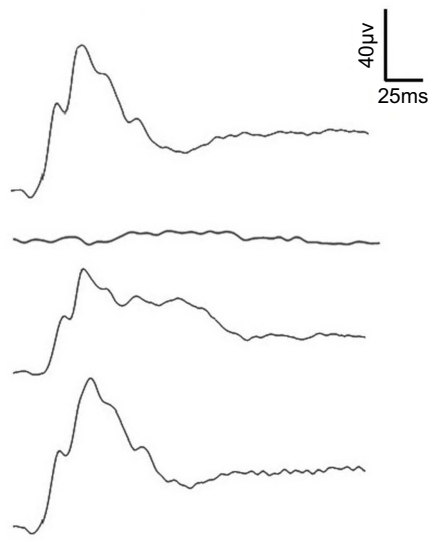

MNU+PBS

B

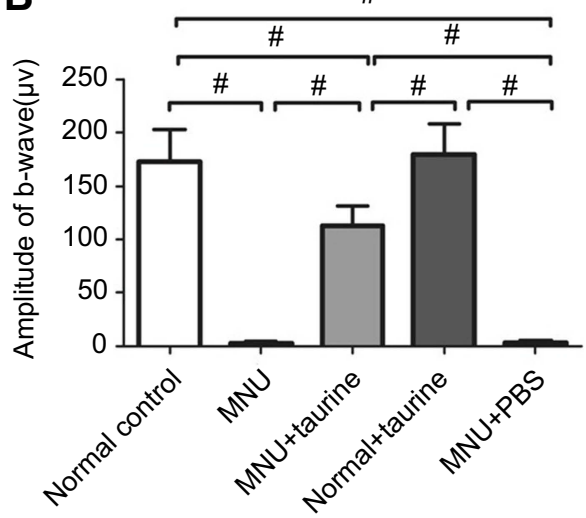

\section{D}

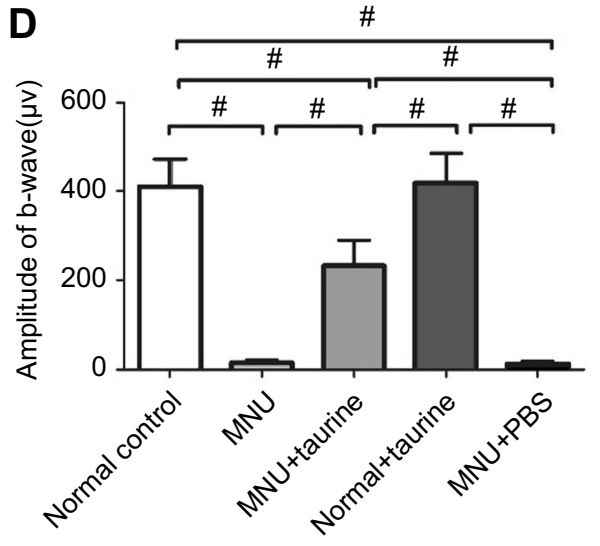

C

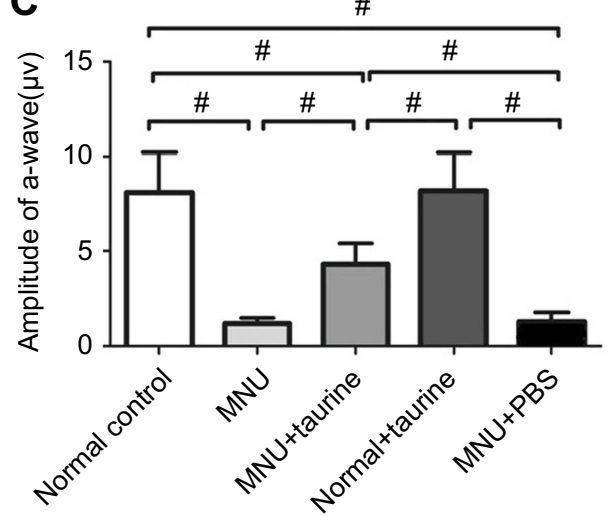

E

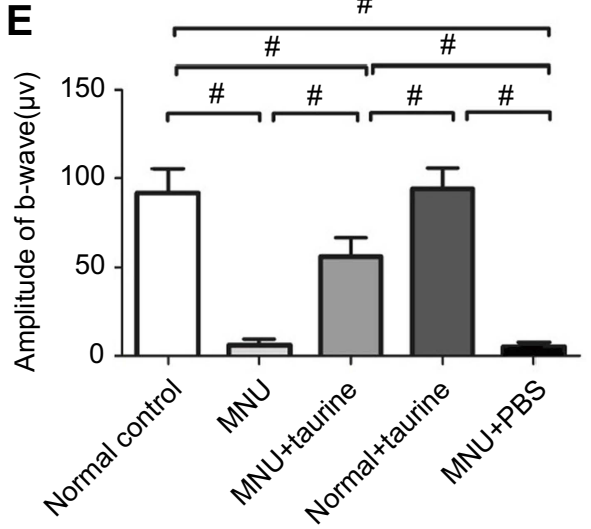

Figure 3 (A) The light stimulus induced large electroretinography (ERG) responses in mice of the normal control group. The ERG responses in the N-methyl-N-nitrosourea (MNU) group were terribly abolished. (B-C) The scotopic and photopic a-wave amplitudes in the MNU+taurine group was significantly larger than those in the MNU group. (D-E) The scotopic and scotopic b-wave amplitudes in the MNU group were significantly smaller compared with the normal control group. Conversely, the scotopic and photopic b-wave amplitudes in the MNU+taurine group were significantly larger than those in the MNU group, suggesting that the taurine treatment prevented the ERG functional reduction in the MNU-administered mice (ANOVA analysis followed by Bonferroni's post-hoc analysis was performed, ${ }^{\#} P<0.01$, for differences between groups; $n=10)$.

$\mathrm{n}=10)$. Conversely, the scotopic and photopic b-wave amplitudes in the MNU+taurine group were significantly larger than those in the MNU group $(P<0.01 ; \mathrm{n}=10)$. The scotopic and photopic b-wave amplitudes in the MNU+taurine group were $56.9 \%$ and $61.3 \%$ of the normal controls, respectively. These data suggested that the taurine treatment prevented 
partially, but significantly, the ERG functional reduction in the MNU-administered mice.

In order to determine whether the aforementioned electrophysiological protection could lead to vision improvements in a behavioral sense, we tested the experimental animals by the vision-guided optokinetic tests. The visual acuity and contrast sensitivity in the MNU group were significantly smaller than those in the group $(P<0.01 . \mathrm{n}=10$; Figure 4$)$. Mice in the $\mathrm{MNU}+$ taurine group responded better to the raster stimulus compared with the MNU group. The visual acuity and contrast sensitivity in the MNU+taurine group were significantly larger than those in the MNU group $(P<0.01 ; \mathrm{n}=10)$. Additionally, the visual acuity and contrast sensitivity in the MNU+PBS group were not significantly different from those in the MNU group ( $P>0.05, \mathrm{n}=10)$. These data suggested the taurine treatment conferred beneficial effects on the optokinetic performance of MNU-administered mice.

\section{Taurine mediated protective effects on cone photoreceptors}

Previous studies have shown that the cone photoreceptors are more sensitive to the taurine depletion than the rods. ${ }^{20}$ As the cones only account for approximately 3\% of the entire crew of photoreceptors, we cannot deduce the cone vitality from indicators such as the ONL thickness. Therefore, the immunostaining works were performed to determine the taurine-mediated effects on the cones. In the retinal sections of normal controls group, PNA staining was evident at the outer segments of cones (Figure 5). PNA staining in retinal sections of MNU-administered group was extremely faint. Conversely, a pronounced proportion of PNA staining was retained in the retinal sections of the MNU+taurine group. Moreover, the PNA staining in the retinal flat mounts was examined. The MNU group showed a significant reduction in the counts of PNA-positive cells compared with the normal control group $(P<0.01 ; n=10)$. The average PNA-positive cell counts in the MNU+taurine group were significantly larger than that in the MNU group $(P<0.01 ; \mathrm{n}=10)$, suggesting that the taurine treatment resulted in a significant increase in the cone vitality. In the retinal whole mounts of MNU+taurine group, PNA-positive cell counts of the inferior-nasal (IN) quadrant were the smallest among the four retinal quadrants. On the other hand, the cone photoreceptors in the superior-temporal (ST) and superior-nasal (SN) quadrants were preferentially preserved by the taurine treatment.

In greater detail, the vitality of different cone populations was examined using opsin-specific antibodies. As shown in the retinal sections, both S-opsin (Figure 6) and M-opsin (Figure 7) staining in the MNU group were lost. On the other hand, the S- and M-opsin staining were evident in the ONL of the MNU+taurine group, although with a decayed manner relative to the normal controls. Moreover, the cone opsin staining in the retinal flat mounts was examined. The $\mathrm{S}$-opsin and M-opsin positive cell counts in the MNU
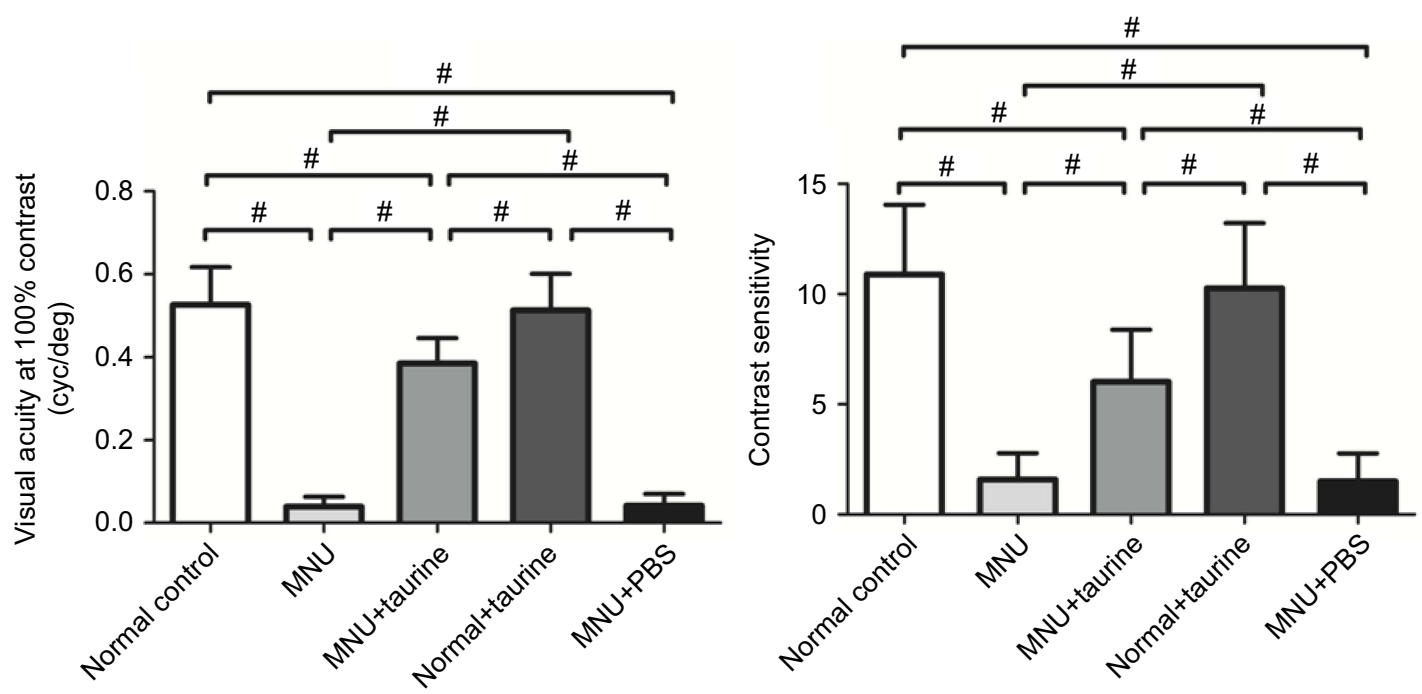

Figure 4 The visual acuity and contrast sensitivity in the $\mathrm{N}$-methyl-N-nitrosourea (MNU) group were significantly smaller than those in the group. Mice in the MNU+taurine group responded better to the raster stimulus compared with the MNU group. The visual acuity and contrast sensitivity in the MNU+taurine group were significantly larger than those in the MNU group, indicating that the taurine treatment conferred beneficial effects on the optokinetic performance of MNU-administered mice (ANOVA analysis followed by Bonferroni's post-hoc analysis was performed, ${ }^{\#} P<0.01$, for differences between groups; $\left.n=10\right)$. 


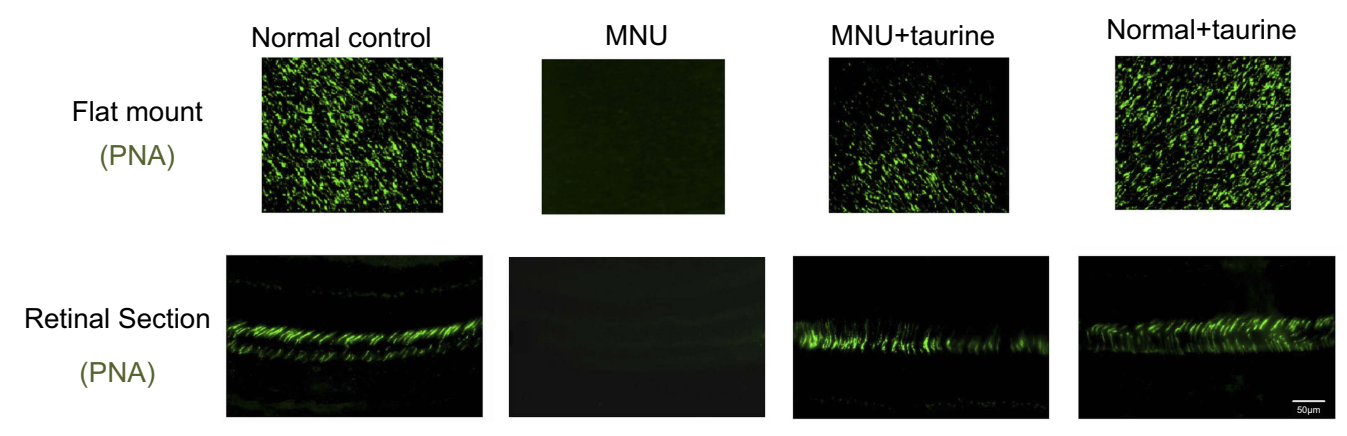

Figure 5 In the retinal sections of normal controls group, PNA staining was evident at the outer segments of cones. A pronounced proportion of PNA staining was retained in the retinal sections of the MNU+taurine group. The average PNA-positive cell counts in the MNU+taurine group were significantly larger than that in the $\mathrm{N}$-methyl-Nnitrosourea (MNU) group. In the retinal whole mounts of MNU+taurine group, PNA-positive cell counts of the IN quadrant were the smallest among the four retinal quadrants. The cone photoreceptors in the ST and SN quadrants were preferentially preserved by the taurine treatment. Abbreviations: ST, superior-temporal; SN, superior-nasal; IT, inferior-temporal; IN, inferior-nasal; C, central.

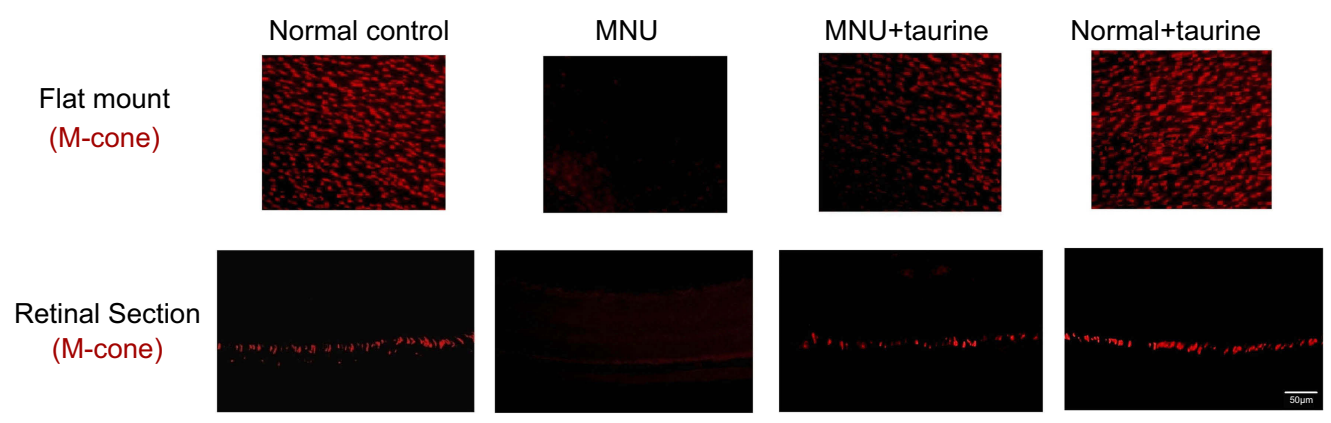

Figure 6 In the retinal sections, S-opsin staining in the N-methyl-N-nitrosourea (MNU) group was lost. On the other hand, the S-opsin staining was evident in the ONL of the $\mathrm{MNU}$ +taurine group. In the retinal flat mounts, the S-cone opsin positive cell counts in the MNU+taurine group were significantly larger than those in the MNU group. In the MNU+taurine group, the S-cone opsin positive cells were distributed throughout the retinal flat mount. Most of the S-opsin positive cells were located in the IN quadrants and the fewest cells in the ST quadrant, suggesting that S-cone photoreceptors from the IN quadrant benefited most from the taurine treatment. Abbreviations: ST, superior-temporal; SN, superior-nasal; IT, inferior-temporal; IN, inferior-nasal; C, central.

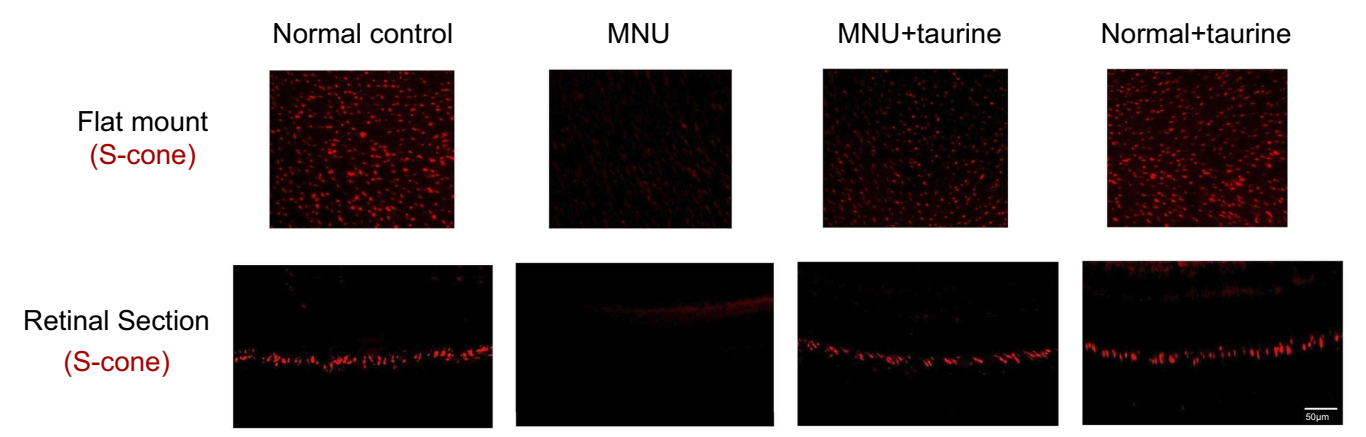

Figure 7 In the retinal sections, $\mathrm{M}$-opsin staining in the $\mathrm{N}$-methyl-N-nitrosourea (MNU) group was undetectable. By contrast, the $\mathrm{M}$-opsin staining was evident in the ONL of the MNU+taurine group. In the retinal flat mounts, the M-cone opsin positive cell counts in the MNU+taurine group were significantly larger than those in the MNU group. In the MNU+taurine group, the M-opsin positive cells were distributed throughout the retinal flat mount. Most of the M-opsin positive cells were located in the ST quadrants and the fewest cells in the IN quadrant, suggesting that M-cone photoreceptors from the ST quadrant benefited most from the taurine treatment. Abbreviations: ST, superior-temporal; SN, superior-nasal; IT, inferior-temporal; IN, inferior-nasal; C, central.

+ taurine group were significantly larger than those in the MNU group $(P<0.01 ; \mathrm{n}=10)$. Comparison analysis showed that the average count of S-and $\mathrm{M}$-opsin positive cells in the MNU+taurine group was, respectively, $40.4 \%$ and of $44.4 \%$ that in the normal control. In the MNU+taurine group, both the S- and M-opsin positive cells were distributed throughout the retinal flat mount. However, the distribution was not uniformly equal and formed a topographic gradient across retina: most of the M-opsin positive cells were located in the ST quadrants and the fewest cells in the IN quadrant, 
whereas most of the S-opsin positive cells were located in the IN quadrants and the fewest cells in the ST quadrant. These findings suggested the $\mathrm{M}$ - and S-opsin cone populations were both amenable to the taurine treatment.

\section{Mechanisms responsible for the protection}

Active caspase- 3 assay was performed to verify the taurineinduced effects on the photoreceptor apoptosis (Figure 8A). There was heavy activated caspase-3 immunostaining throughout the retinal sections of MNU group. Conversely, retinal sections from the MNU+taurine group had relatively lower activated caspase-3 immunostaining. These results suggested that taurine treatment could suppress the caspase- 3 activation in the MNU-administered mice. Moreover, the qRTPCR was performed to analyze the expression levels of several apoptotic factors. In the MNU+taurine group, the mRNA levels of Caspase-3, Calpain-2, and Bax were significantly down-regulated compared with the MNU group $(P<0.01$; $\mathrm{n}=10$ ). On the other hand, the mRNA level of Bcl-2 in the MNU+taurine group was significantly up-regulated than that in the MNU group $(P<0.05 ; \mathrm{n}=10$; Figure $8 \mathrm{~B}-\mathrm{E})$. These findings suggested that the anti-apoptotic mechanism was, at least partly, responsible for the taurine-mediated protection. Moreover, the retinal status of MDA, a stable metabolite of lipid peroxidation, in the MNU group was distinctly higher compared with the normal control group. The retinal concentration of MDA in the MNU+taurine group was significantly lower than that in the MNU group $(P<0.01$; $n=10$; Figure $8 \mathrm{~F})$, suggesting the taurine treatment could alter the retinal oxidation level of MNU administrated mice. The expression level of SOD, an endogenous antioxidant, in the MNU+taurine group was significantly higher than that in the MNU group $(P<0.01$;

A

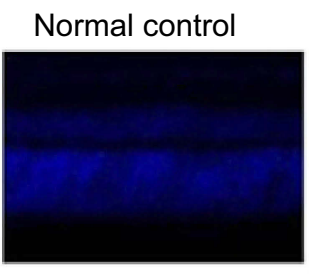

B

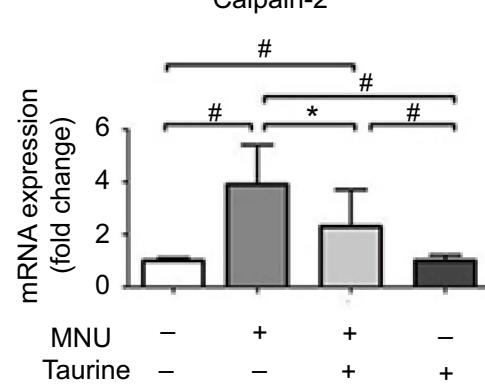

MNU

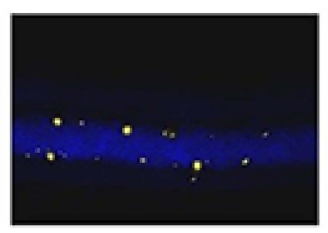

MNU+taurine

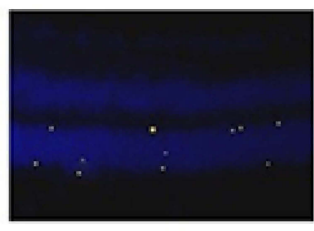

Normal+taurine

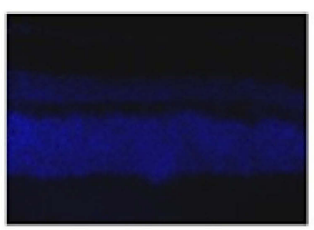

C

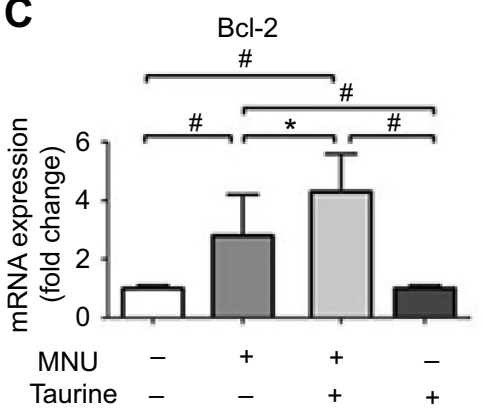

D

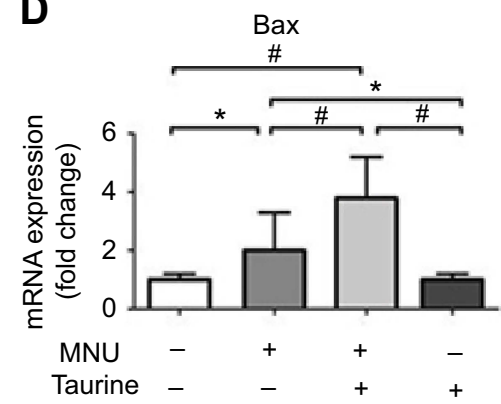

E

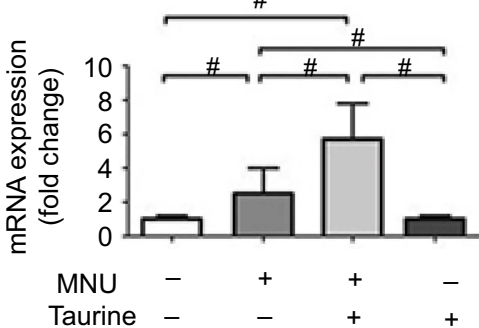

$\mathbf{F}$

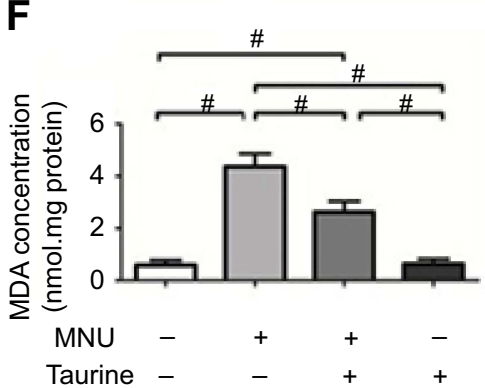

G

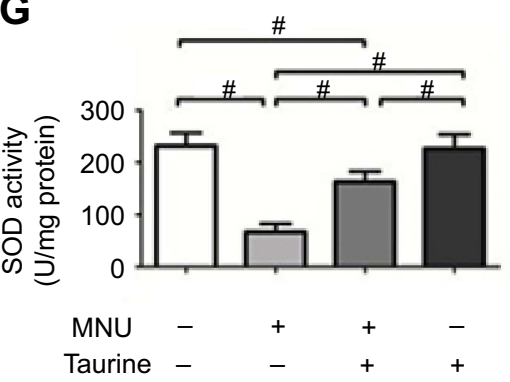

Figure 8 (A) Active caspase-3 assay was performed to verify the taurine-induced effects on the photoreceptor apoptosis. There was heavy activated caspase-3 immunostaining throughout the outer nuclear layer (ONL) of MNU group. Conversely, retinas from MNU+taurine group had relatively lower activated caspase-3 immunostaining. (B-E) The qRT-PCR was performed to analyze the expression levels of several apoptotic factors. In the MNU+taurine group, the mRNA levels of Calpain-2, Caspase-3, and Bax were significantly down-regulated compared with the MNU group. On the other hand, the mRNA level of Bcl-2 in the MNU+taurine group was significantly up-regulated than that in the MNU group. (F) The retinal status of MDA in the MNU group was distinctly higher compared with the normal control group. The retinal concentration of malondialdehyde(MDA) in the MNU+taurine group was significantly lower than that in the MNU group. (G) The expression level of superoxide dismutase(SOD) in the MNU+taurine group was significantly higher than that in the MNU group $(P<0.01$; $n=10)$, suggesting the taurine treatment could enhance the antioxidant ability of MNU-administrated mice (ANOVA analysis followed by Bonferroni's post-hoc analysis was performed, ${ }^{*} P<0.05$, ${ }^{*} P<0.0 \mathrm{I}$, for differences between groups; $n=10)$. 
$\mathrm{n}=10$; Figure $8 \mathrm{G}$ ), suggesting the taurine treatment could enhance the antioxidant ability of MNU-administrated mice.

\section{Discussion}

RP can lead to progressive photoreceptor loss and severe visual impairments in the subjects. At least three different modes of inheritance and 100 gene mutations have been implicated in etiology. ${ }^{4,21}$ The overall prognosis remains dismal due to the heterogeneous pathogenesis, multiple risk factors, and long cycle of intervention. Even for these affected family members presumably sharing the mutual causative gene mutation, their progressive rate and pathological outcome are subjected to considerable variations. Therefore, it is feasible to build a therapeutic strategy on the basis of molecules with antiapoptotic properties. ${ }^{3,22}$ In this study, we explored the potential beneficial effects of taurine on the photoreceptor degeneration. Typically, MNU toxicity induces massive photoreceptor degeneration in mice retina. In MNU-administered mice, the ONL is disrupted and the retina is drastically thinned. Taurine is highly concentrated (more than $60 \%$ ) in the ONL of the vertebrate retina. ${ }^{12}$ Accordingly, retinal taurine level in the MNU-administered mice decreases compared with normal mice. On the other hand, the plasmatic taurine in MNUadministered mice (without exogenous taurine administration) mainly comes from dietary supplements, and the MNU is unlikely to affect the intestinal absorption of taurine into blood circulation. This should be the reason for the decreased retinal taurine level but relatively normal plasmatic level in MNU-administered mice. Herein, we show that intravenous delivery of taurine was able to alleviate the photoreceptor degeneration and visual impairments in the MNUadministered mice. Compared with the untreated controls, relatively intact retinal architectures with thicker ONL were retained in the taurine-treated animals. In particular, the taurine-treated mice exhibited elevated levels of cone photoreceptor viability. Traditionally, taurine is administered as a dietary supplement in therapeutic trials. ${ }^{23-25}$ Dietary taurine could be absorbed into the circulation system, whereas this process is highly dependent on the taurine transporters in intestinal epithelium. Herein, we show that the directly intravenous delivery can act as an efficient approach to enhance the retinal taurine level. Of note, the following improvements are integrated into the methodology of our work. First, the in vivo examination approaches are used to evaluate the efficacy of treatment. For instance, SD-OCT examination can measure the retinal thicknesses noninvasively without sacrificing the animals. ${ }^{26}$ Moreover, previous therapeutic trials only describe the vitality of entire photoreceptors, potentially obscuring accurate information for a single photoreceptor population. Herein, we dissect the taurine-induced effects on the rod and cone photoreceptors, respectively. Despite the pathophysiology of MNU-induced model is somewhat different from that in RP patients, our laboratory findings lay the groundwork for future application of taurine in clinical practice.

As a nutritional amino acid, taurine is well known for its vital contribution to neuron development and survival. Retinal taurine is provided by retinal pigment epithelium cells and Müller cells, which normally take up taurine and pass it on to photoreceptors. ${ }^{27}$ Photoreceptor cells need an adequate supply of extracellular taurine and depend upon their own transporter for osmoregulation. ${ }^{28}$ The taurine depletion will cause RGC and cone photoreceptor death. ${ }^{29,30}$ On the other hand, taurine supplementation may help to prevent retinal degeneration, especially those that commence with S-cone degeneration. ${ }^{31,32}$ Previous studies show that taurine may act as functional trigger of cones, and at higher amount so it would have altered retina physiology. ${ }^{12,33}$ Moreover, taurine acts as a neurotransmitter via specific receptors and interferes with functions of other transmitter systems. ${ }^{33,34}$ For instance, taurine can activate the $\mathrm{GABA}_{\mathrm{B}}$ receptor and promote RGCs survival, thereby modifying the progress rate of retinal degeneration. ${ }^{35}$ Taurine and glutamate are reciprocally inhibiting each other, thereby contributing to fine-tuning visual signals in the retinal circuits. ${ }^{36}$ Taurine is also a potential neuromodulator in the process of intracellular calcium regulation. Taurine can regulate the transmembrane movements of calcium via the voltage-gated $\mathrm{Ca}^{2+}$ channels and the NMDA glutamate receptors. ${ }^{37,38}$ Since the retinal taurine is maximally concentrated in the ONL, researchers propose that the taurine deficiency should be taken into account whenever photoreceptor degeneration is detected under pathological conditions. $^{39,40}$ A significant decreased retinal taurine level has been reported in RCS rats and was closely correlated to the photoreceptor degeneration. ${ }^{41} \mathrm{RP}$ patients also have decreased levels of taurine uptake into platelets and lower blood taurine levels compared with healthy population. $^{42,43}$ In this study, we show that the MNU toxicity induced a prominent taurine deficiency in mouse retina. These findings agree well and highlight the possibility that exogenous taurine might exert beneficial effects on the photoreceptors. Our immunostaining results suggest that taurine therapy rescues the $\mathrm{M}$ - and $\mathrm{S}$-cones, which are 
in charge of the high visual acuity and color discrimination, respectively. In RP pathogenesis, the etiological mutations affect exclusively the rod-specific genes, but the primary rod apoptosis is often followed by the cone degeneration. ${ }^{3,21}$ Actually, the main clinical vision impairment that affects patient's daily life should be attributed to the secondary cone degeneration rather than the rod death. As long as the cones are preserved, patients would function very well in bright circumstance and carry on relatively normal lives despite the rods loss. ${ }^{44-46}$ Our findings indicate that taurine is able to overcome MNU-induced photoreceptor degeneration in mice. Admittedly, the cone circuitry in mice is different from fovea dependence on cones in human. The rodent retinal has a cone distribution primarily as a ring in the equatorial retina which is quite different from the fovea dependence on cones in human. ${ }^{47}$ Therefore, the taurine-induced beneficial effects on cones should be further validated in the large animal model (such as primate) which has retinal architectures more similar to the human. ${ }^{48}$ In clinical settings, taurine has been enrolled into the dietary anti-oxidant regiments to combat against RP and age-related macular degeneration. However, the protective effect was not absolutely satisfactory. ${ }^{49,50}$ As highly water soluble, taurine intake from dietary sources is highly dependent on specific transporter expression in intestines. Dietary taurine must first be transported across the intestinal barrier to reach the blood. ${ }^{51}$ A substantial portion of the orally delivered taurine cannot diffuse across intestinal barrier to reach the blood. Accordingly, the intravascular or intraocular delivery might act as a more promising approach to ensure higher concentration of taurine in the retina.

Apoptosis is considered as the ultimately mutual pathway leading to photoreceptor death. ${ }^{9,10}$ TUNNEL assay is a reliable method to identify the apoptotic cells and quantify the apoptotic levels in retinas. The TUNEL results show that taurine is capable of ameliorating the photoreceptor apoptosis in the MNU-administered mice. This notion is further reinforced by the fact that taurine modulates the mRNA levels of apoptotic and anti-apoptotic factors in retinas. The mRNA level of Caspase-3, a pivotal mediator of cell apoptosis, is significantly reduced by taurine. On the other hand, taurine is able to enhance the Bcl-2 expressions and adjust the Bcl/Bax ratio toward a net "anti-apoptotic" effect. Additionally, the expression level of Calpain-2, a calcium-dependent cysteine protease, is down-regulated by taurine. It has been shown that taurine could reduce calcium overload in RGCs and brain neurons via an intracellular calpain-dependent pathway. ${ }^{52}$ Taurine also reduces intracellular calcium levels by inhibiting the $\mathrm{Ca}^{2+}$ influx through voltage-gated channels. ${ }^{37}$ Consequently, taurine might alleviate the calcium overload which acts as an initiator in photoreceptor apoptosis. Moreover, there is a growing consensus that the taurine-induced protective effect is correlated with its antioxidant activity. ${ }^{12,14}$ Taurine can stabilize the electron transport chain and suppress the ROS generation. Its osmotic properties could also limit the plasma membrane permeabilization, thereby alleviating the deleterious effect of mitochondrial dysfunction. ${ }^{53}$ Herein, we show that the retinal SOD level in the MNU-administered mice is increased by taurine, whereas the retinal MDA level is decreased. The SOD system is among the most critical and ubiquitous oxidation enzymes that are complicated tuned in the retina. These findings indicate that taurine can bolster the endogenous antioxidants and counteract the oxidative damage in retina. Admittedly, the etiology of the MNU-induced photoreceptor degeneration is different from that occurs in models of RP, although the pathologic outcomes are somewhat similar. Moreover, the MNU-induced photoreceptor degeneration is rapidly progressing. However, the RP is a relatively chronic retinopathy in human patients. Therefore, although the outcome of the MNU administration partially resembles RP (ie, widespread rod death), the mechanistic underpinning and kinetics are very different. These considerations are crucial in any pharmacological study which proposes clinical testing of taurine.

\section{Conclusion}

The taurine deficiency occurs during the MNU-induced photoreceptor degeneration. Intravenous delivery of taurine inhibits effectively the photoreceptor loss and visual impairments in the MNU-administered mice. Taurine affords these protective effects by modulating apoptosis and alleviating oxidative stress in degenerative retina. These findings indicate that systemic taurine administration may act as a promising therapeutic potion for degenerative retinopathy.

\section{Acknowledgments}

This study is supported in part by the National Key Research and Development Plan of China [No. 2018YFA0107303] and National Natural Science Foundation of China [No. 81600767]. 


\section{Disclosure}

The authors report no conflicts of interest in this work.

\section{References}

1. Falsini B, Iarossi G, Chiaretti A, et al. NGF eye-drops topical administration in patients with retinitis pigmentosa, a pilot study. $J$ Transl Med. 2016;14:8. doi:10.1186/s12967-016-0867-z

2. Hartong DT, Berson EL, Dryja TP. Retinitis pigmentosa. Lancet. 2006;368:1795-1809. doi:10.1016/S0140-6736(06)69740-7

3. Guadagni V, Novelli E, Piano I, Gargini C, Strettoi E. Pharmacological approaches to retinitis pigmentosa: A laboratory perspective. Prog Retin Eye Res. 2015;48:62-81. doi:10.1016/j. preteyeres.2015.06.005

4. Sorrentino FS, Gallenga CE, Bonifazzi C, Perri P. A challenge to the striking genotypic heterogeneity of retinitis pigmentosa: a better understanding of the pathophysiology using the newest genetic strategies. Eye. 2016;30:1542-1548. doi:10.1038/eye.2016.197

5. Tsubura A, Yoshizawa K, Kuwata M, Uehara N. Animal models for retinitis pigmentosa induced by $\mathrm{MNU}$; disease progression, mechanisms and therapeutic trials. Histol Histopathol. 2010;25:933-944. doi:10.14670/HH-25.933

6. Reisenhofer MH, Balmer JM, Enzmann V. What can pharmacological models of retinal degeneration tell us? Curr Mol Med. 2017;17:100-107. doi:10.2174/1566524017666170331162048

7. Nomura-Komoike K, Saitoh F, Komoike Y, Fujieda H. DNA damage response in proliferating müller glia in the mammalian retina. Invest Ophthalmol Vis Sci. 2016;57:1169-1182. doi:10.1167/iovs.15-18101

8. Emoto Y, Yoshizawa K, Kinoshita Y, Yuki M, Yuri T, Tsubura A. Susceptibility to N-methyl-N-nitrosourea-induced retinal degeneration in different rat strains. $J$ Toxicol Pathol. 2016;29:67-71. doi:10.1293/tox.2015-0062

9. Comitato A, Sanges D, Rossi A, Humphries MM, Marigo V. Activation of Bax in three models of retinitis pigmentosa. Invest Ophthalmol Vis Sci. 2014;13(55):3555-3562. doi:10.1167/iovs.14-13917

10. Sanvicens N, Cotter TG. Ceramide is the key mediator of oxidative stress-induced apoptosis in retinal photoreceptor cells. $J$ Neurochem. 2006;98:1432-1434. doi:10.1111/j.1471-4159.2006.03977.x

11. Tao Y, Geng L, Wang L, et al. Use of hydrogen as a novel therapeutic strategy against photoreceptor degeneration in retinitis pigmentosa patients. Med Sci Monit. 2016;22:776-779.

12. Ripps H, Shen W. Review: taurine: a "very essential" amino acid. Mol Vis. 2012;18:2673-2686.

13. Takatani $\mathrm{T}$, Takahashi $\mathrm{K}$, Uozumi $\mathrm{Y}$, et al. Taurine prevents the ischemia-induced apoptosis in cultured neonatal rat cardiomyocytes through Akt/caspase-9 pathway. Biochem Biophys Res Commun. 2004;316:484-489. doi:10.1016/j.bbrc.2004.02.066

14. Froger N, Moutsimilli L, Cadetti L, et al. Taurine: the comeback of a neutraceutical in the prevention of retinal degenerations. Prog Retin Eye Res. 2014;41:44-63. doi:10.1016/j.preteyeres.2014.03.001

15. Froger N, Cadetti L, Lorach H, et al. Taurine provides neuroprotection against retinal ganglion cell degeneration. PLoS One. 2012;7: e42017. doi:10.1371/journal.pone.0042017

16. Lake N, Verdone-Smith C. Immunocytochemical localization of taurine in the mammalian retina. Curr Eye Res. 1989;8:163-173.

17. Pasantes-Morales H, Domínguez L, Campomanes MA, Pacheco P. Retinal degeneration induced by taurine deficiency in light-deprived cats. Exp Eye Res. 1986;43:55-60.

18. Hayes KC, Carey RE, Schmidt SY. Retinal degeneration associated with taurine deficiency in the cat. Science. 1975;188:949-951.

19. Kretschmer F, Sajgo S, Kretschmer V. A system to measure the Optokinetic and Optomotor response in mice. J Neurosci Methods. 2015;256:91-105. doi:10.1016/j.jneumeth.2015.08.007
20. Gaucher D, Arnault E, Husson Z, et al. Taurine deficiency damages retinal neurones: cone photoreceptors and retinal ganglion cells. Amino Acids. 2012;43:1979-1993. doi:10.1007/s00726-012-1273-3

21. Petit L, Punzo C. mTORC1 sustains vision in retinitis pigmentosa. Oncotarget. 2015;6:16786-16787. doi:10.18632/oncotarget.4466

22. Cottet S, Schorderet DF. Mechanisms of apoptosis in retinitis pigmentosa. Curr Mol Med. 2009;9:375-383.

23. Figueroa AL, Figueiredo H, Rebuffat SA, Vieira E, Gomis R. Taurine treatment modulates circadian rhythms in mice fed A high fat diet. Sci Rep. 2016;6:36801. doi:10.1038/srep36801

24. Abd Elwahab AH, Ramadan BK, Schaalan MF, Tolba AM. A novel role of SIRT1/FGF-21 in taurine protection against cafeteria diet-induced steatohepatitis in rats. Cell Physiol Biochem. 2017;43:644-659. doi:10.1159/000480649

25. Rosa FT, Freitas EC, Deminice R, Jordão AA, Marchini JS. Oxidative stress and inflammation in obesity after taurine supplementation: a double-blind, placebo-controlled study. Eur $J$ Nutr. 2014;53:823-830. doi:10.1007/s00394-013-0586-7

26. Kashani AH, Chen CL, Gahm JK, et al. Optical coherence tomography angiography: A comprehensive review of current methods and clinical applications. Prog Retin Eye Res. 2017;60:66-100. doi:10.1016/j.preteyeres.2017.07.002

27. Rascher K, Servos G, Berthold G, et al. Light deprivation slows but does not prevent the loss of photoreceptors in taurine transporter knockout mice. Vision Res. 2004;44:2091-2100. doi:10.1016/j. visres.2004.03.027

28. Schaffer S, Takahashi K, Azuma J. Role of osmoregulation in the actions of taurine. Amino Acids. 2000;19(3-4):527-546.

29. Hadj-Saïd W, Froger N, Ivkovic I, et al. Quantitative and topographical analysis of the losses of cone photoreceptors and retinal ganglion cells under taurine depletion. Invest Ophthalmol Vis Sci. 2016;57:4692-4703. doi:10.1167/iovs.16-19535

30. Jammoul F, Dégardin J, Pain D, et al. Taurine deficiency damages photoreceptors and retinal ganglion cells in vigabatrin-treatedneonatal rats. Mol Cell Neurosci. 2010;43:414-421. doi:10.1016/j. men.2010.01.008

31. García-Ayuso D, Di Pierdomenico J, Hadj-Said W, et al. Taurine depletion causes ipRGC loss and increases light-induced photoreceptor degeneration. Invest Ophthalmol Vis Sci. 2018;59:1396-1409. doi:10.1167/iovs.17-23258

32. Trouillet A, Dubus E, Dégardin J, et al. Cone degeneration is triggered by the absence of USH1 proteins but prevented by antioxidant treatments. Sci Rep. 2018;8(1):1968. doi:10.1038/s41598-018-20171-0

33. Oja SS, Saransaari P. Pharmacology of taurine. Proc West Pharmacol Soc. 2007;50:8-15.

34. Fletcher EL, Kalloniatis M. Neurochemical development of the degenerating rat retina. J Comp Neurol. 1997;388:1-22.

35. Hadj-Saïd W, Fradot V, Ivkovic I, Sahel JA, Picaud S, Froger N. Taurine promotes retinal ganglion cell survival through $\mathrm{GABA}_{\mathrm{B}}$ receptor activation. Adv Exp Med Biol. 2017;975 Pt 2:687-701. doi:10.1007/978-94-024-1079-2 54

36. Bulley S, Shen W. Reciprocal regulation between taurine and glutamate response via $\mathrm{Ca} 2+-$ dependent pathways in retinal third-order neurons. J Biomed Sci. 2010;17 Suppl1:S5. doi:10.1186/1423-0127-17-S1-S5

37. El Idrissi A. Taurine increases mitochondrial buffering of calcium: role in neuroprotection. Amino Acids. 2008;34:321-328. doi:10.1007/ s00726-006-0396-9

38. Wu JY, Wu H, Jin Y, et al. Mechanism of neuroprotective function of taurine. Adv Exp Med Biol. 2009;643:169-179. doi:10.1007/9780-387-75681-3_17

39. Froger N, Jammoul F, Gaucher D, et al. Taurine is a crucial factor to preserve retinal ganglion cell survival. Adv Exp Med Biol. 2013;775:69-83. doi:10.1007/978-1-4614-6130-2_6

40. Huxtable RJ. Taurine in the central nervous system and the mammalian actions of taurine. Prog Neurobiol. 1989;32:471-533. 
41. Schmidt SY, Aguirre GD. Reductions in taurine secondary to photoreceptor loss in Irish setters with rod-cone dysplasia. Invest Ophthalmol Vis Sci. 1985;26:679-683.

42. Airaksinen EM, Sihvola P, Airaksinen MM, Sihvola M, Tuovinen E. Uptake of taurine by platelets in retinitis pigmentosa. Lancet. 1979;1:474-475.

43. Arshinoff SA, McCulloch JC, Macrae W, Stein AN, Marliss EB. Amino acids in retinitis pigmentosa. $\mathrm{Br} J$ Ophthalmol. 1981;65:626-630. doi:10.1136/bjo.65.9.626

44. Narayan DS, Wood JP, Chidlow G, Casson RJ. A review of the mechanisms of cone degeneration in retinitis pigmentosa. Acta Ophthalmol. 2016;94:748-754. doi:10.1111/aos.13141

45. Friedburg C, Serey L, Sharpe LT, Trauzettel-Klosinski S, Zrenner E. Evaluation of the Night Vision Spectacles on patients with impaired night vision. Graefes Arch Clin Exp Ophthalmol. 1999;237:125-3635.

46. Tao Y, Chen T, Yang GQ, et al. Anthocyanin can arrest the cone photoreceptor degeneration and act as a novel treatment for retinitis pigmentosa. Int $J$ Ophthalmol. 2016;9:153-158. doi:10.18240/ ijo.2016.01.25

47. Szél A, Röhlich P, Caffé AR, van Veen T. Distribution of cone photoreceptors in the mammalian retina. Microsc Res Tech. 1996;35 (6):445-462. doi:10.1002/(SICI)1097-0029(19961215)35:6<445:: AID-JEMT4>3.0.CO;2-H
48. Viets K, Eldred K, Johnston RJ Jr. Mechanisms of photoreceptor patterning in vertebrates and invertebrates. Trends Genet. 2016;32 (10):638-659. doi:10.1016/j.tig.2016.07.004

49. Pasantes-Morales H, Quiroz H, Quesada O. Treatment with taurine, diltiazem, and vitamin $\mathrm{E}$ retards the progressive visual fieldreduction in retinitis pigmentosa: a 3-year follow-up study. Metab Brain Dis. 2002;17(3):183-197.

50. Cangemi FE. TOZAL Study: an open case control study of an oral antioxidant and omega-3 supplement for dry AMD. BMC Ophthalmol. 2007;7:3. doi:10.1186/1471-2415-7-3

51. Anderson CM, Howard A, Walters JR, Ganapathy V, Thwaites DT. Taurine uptake across the human intestinal brush-border membrane is via two transporters: $\mathrm{H}+$-coupled PAT1 (SLC36A1) and $\mathrm{Na}+-$ and $\mathrm{Cl}$ (-)-dependent TauT (SLC6A6). J Physiol. 2009;587(Pt 4):731-744. doi:10.1113/jphysiol.2008.164228

52. Sun $\mathrm{M}, \mathrm{Xu}$ C. Neuroprotective mechanism of taurine due to up-regulating calpastatin and down-regulating calpain and caspase-3 during focal cerebral ischemia. Cell Mol $N$ Eurobiol. 2008;28:593-611. doi:10.1007/s10571-007-9183-8

53. Lambert IH, Kristensen DM, Holm JB, Mortensen OH. Physiological role of taurine-from organism to organelle. Acta Physiol. 2015;213:191-212. doi:10.1111/apha.12365
Drug Design, Development and Therapy

\section{Publish your work in this journal}

Drug Design, Development and Therapy is an international, peerreviewed open-access journal that spans the spectrum of drug design and development through to clinical applications. Clinical outcomes, patient safety, and programs for the development and effective, safe, and sustained use of medicines are a feature of the journal, which has also

\section{Dovepress}

been accepted for indexing on PubMed Central. The manuscript management system is completely online and includes a very quick and fair peer-review system, which is all easy to use. Visit http://www. dovepress.com/testimonials.php to read real quotes from published authors. 